\title{
Impacto económico del efecto de la contaminación del aire en la salud: Managua- Nicaragua (años 1996-2001)
}

\section{Carlos Antonio Narváez Silva* y Octavio Javier Martinez Baltodano**}

\section{Recibido: noviembre de 2010 / Aceptado: febrero de 2011}

Existe una relación positiva entre la contaminación del aire por partículas menores a diez micrómetros $\left(\mathrm{PM}_{10}\right)$, partículas totales suspendidas (PTS) y ozono $\left(\mathrm{O}_{3}\right)$, y la tasa de morbilidad en Managua de (4\%, $8 \%$ y 30\%) respectivamente. Los resultados del monitoreo de calidad del aire realizado por la Universidad Nacional de Ingeniería por medio del Centro de Investigaciones en Medio Ambiente, muestran que las $\mathrm{PM}_{10}$ y las PTS están fuera de los límites permisibles establecidos por la organización mundial de la salud (OMS), la Agencia de Protección Ambiental (EPA). Las personas con mayor afectación son los menores de 15 años y los mayores de 60 . Esto provoca un gasto en salud pública en actividades curativas equivalente a 73 C\$ por persona. De acuerdo a informes del Ministerio de Salud, anualmente llegan en promedio 30 mil casos por infecciones respiratorias agudas. Si usamos el 30\% de la relación de PTS con la tasa de morbilidad, tendríamos 10 mil casos causados por la contaminación del aire. Desde 2001 no existe un programa de monitoreo de calidad del aire. Se necesitan 600 mil córdobas anuales para activar el programa y permitirnos aplicar la legislación 217. En nuestro estudio proponemos que de los 120 millones de córdobas anuales que recibe el sector transporte en subsidio, se le reste el daño ambiental que éste provoca a la población por contaminación del aire. Esto aportaría básicamente los 600 mil córdobas que se necesitan para activar el 
programa de calidad del aire. Esta política tendría un impacto de $1.60 \mathrm{C} \$ /$ día por bus (menos de un pasajero al día). La segunda alternativa es gravar un impuesto al consumo de diesel de 10 centavos de córdoba una vez al año; preferiblemente en el mes de mayor consumo. Tomando como base las ventas de diesel, se recaudarían 4 millones de córdobas aproximadamente, dinero suficiente para el proyecto de calidad del aire y para satisfacer la demanda en medicamentos en los centros de salud en Managua.

Palabras clave: contaminación del aire / morbilidad / gasto en salud / instrumento económico

\section{Introducción}

Uno de los principales servicios que provee el medio ambiente a la sociedad es la provisión de soporte de vida, pero éste puede verse afectado por los problemas de contaminación del aire, haciendo que las personas se vuelvan más sensibles a adquirir infecciones respiratorias agudas. Éstas se pueden presentar en términos de crecimiento en las tasas de morbilidad y mortalidad de la población. Por lo tanto podemos afirmar que los problemas de contaminación del aire afectan las condiciones de desarrollo económico en una sociedad debido a que disminuyen la utilidad de los individuos. La contaminación del aire es una amenaza aguda, acumulativa y crónica para la salud humana, un enorme reto por resolver.

¿Cuáles son los costos sociales y ambientales de la contaminación atmosférica? ¿Cuáles son, en especial, los costos de la contaminación atmosférica en términos de morbilidad? Estas y otras preguntas relacionadas con economía y medio ambiente se han convertido en una preocupación fundamental de las agencias internacionales, los gobiernos y los agentes privados en todo el mundo. En Nicaragua es incipiente la investigación cuantitativa que mida económicamente el deterioro y la contaminación. Asimismo, no se han establecidos mecanismos vía instrumentos económicos que permitan un desarrollo más eficiente de la política ambiental establecida en el país.

Dada la usual separación entre los costos privados de los contaminadores y los costos sociales derivados de la contaminación, la eficiencia económica requiere la internalización de los costos sociales (en los costos privados) por medio de instrumentos económicos. Por tanto, el gobierno debe estimar de alguna forma los costos de la contaminación. Esta es claramente una responsabilidad mayor, pues una subestimación de los costos podría impedir una reconversión hacia tecnologías más limpias, y una sobreestimación podría lograr que algunos sectores productivos dejen de ser económicamente viables.

La contaminación del aire principalmente por partículas de polvo, cenizas u hollín dispersas en la atmosfera y cuyo diámetro está entre 2.5 y 10 micrones $\left(\mathrm{PM}_{10}\right)$, y la acumulación de partículas de diferentes diámetros en la atmosfera, mejor conocido como material particulado (PTS) es muy perjudicial para la salud. Muchos estudios epidemiológicos muestran que estos contaminantes ocasionan: ataques de 
asma, síntomas respiratorios y disminuciones del funcionamiento pulmonar en las personas. Por lo tanto, es necesario cuantificar el impacto económico que causan los altos niveles de concentraciones de $\mathrm{PM}_{10}$ y PTS con el objetivo de mostrar los posibles beneficios de controlar y mejorar los niveles de contaminación del aire. Es importante proporcionar evidencia empírica al proveer un marco socioeconómico de los hogares afectados por el problema de la contaminación del aire, ya que es necesario que exista una justificación económica para la realización de programas de monitoreo de aire.

Este estudio se propone contribuir a la problemática planteada con una estimación del impacto de la contaminación atmosférica en la salud. Valorando el costo de estas enfermedades, se realiza un ejercicio de estimación del costo social de la morbilidad inducida por la contaminación atmosférica y de cuánto cuesta reactivar el proyecto de monitoreo de la calidad del aire en Managua junto al mecanismo para sostenerlo por medio de un impuesto a los que causan la contaminación. De igual manera, se valora cómo crear los fondos y satisfacer la demanda de medicamentos de las personas afectadas por la contaminación para que en los centros de salud exista un stock disponible para tratamientos preventivos y curativos.

Para la realización de este estudio se cuenta con información de monitoreo de la calidad del aire: datos de 1996 a 2001. En esos años se tenían seis redes de estaciones que monitoreaban los siguientes contaminantes: partículas menores a diez micrómetros $\left(\mathrm{PM}_{10}\right)$, partículas totales suspendida (PTS), dióxido de nitrógeno $\left(\mathrm{NO}_{2}\right)$, ozono $\left(\mathrm{O}_{3}\right)$, polvos, plomo atmosférico y monóxido de carbono. Los nombres con que se identifican las diferentes estaciones de muestreo son: MARENA, Linda Vista, UNI, Colonia Centro América, Villa Libertad y Siete Sur.

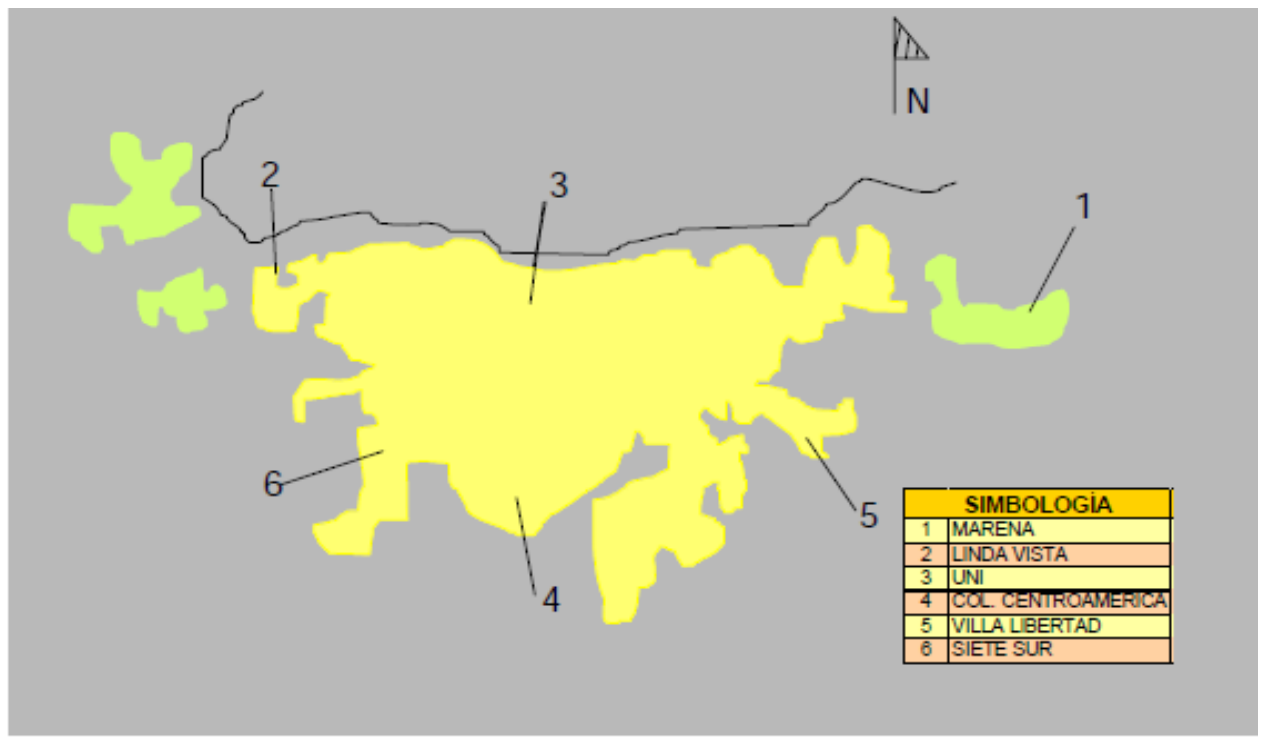

Ilustración 1. Sitios de monitoreo del proyecto aire puro (1996-2001)

Fuente: Universidad Nacional de Ingeniería- Centro de Investigaciones en Medio Ambiente (UNICIEMA, 2001) 
Los resultados del monitoreo indican que las concentraciones de $\mathrm{PM}_{10}$ se encontraban por encima de los valores guías establecidos por la Organización Mundial de la Salud (OMS). Esto implica un mayor riesgo para la salud de los habitantes y una reducción en la calidad de vida, especialmente para los que habitan en los puntos de mayor concentración de contaminación. Por tanto podemos decir que los problemas de salud causados por la contaminación pueden verse influidos por factores tales como magnitud, alcance y duración de exposición, edad, susceptibilidad de cada persona, entre otros.

Desde 1989 hasta 1994 se han realizados en la ciudad de Managua muestreos de la calidad del aire -como los llevados a cabo por el Instituto Nicaragüense de Estudios Territoriales (INETER) y los realizados por las universidades: Nacional de Ingeniería (UNI) de Nicaragua (UNI) y Nacional de Heredia de Costa Rica (UNA) en conjunto con INETER. En dichos muestreos se determinó la presencia de sustancias como: dióxido de azufre $\left(\mathrm{SO}_{2}\right)$, dióxido de nitrógeno $\left(\mathrm{NO}_{2}\right)$, ozono $\left(\mathrm{O}_{3}\right)$, cloro $\left(\mathrm{Cl}_{2}\right)$, monóxido de carbono (CO) y material particulado suspendido (PTS). Los resultados de las mediciones efectuadas en 1994 entre UNI-UNA indicaron elevados niveles tanto de CO como de TPS.

En 1995 se firmó el Convenio de Colaboración entre la UNI y el Programa Ecológico para Centro América (ProEco) a fin de dar inicio al Programa de Monitoreo de la Calidad del Aire de la ciudad de Managua, donde se realizaron muestreos de los siguientes parámetros: $\mathrm{PM}_{10}, \mathrm{NO}_{2}, \mathrm{O}_{3}$, polvos solubles e insolubles, acidez de la lluvia y monóxido de carbono.

La medición de $\mathrm{PM}_{10}$ se realiza utilizando filtros de PTFE, muestreador especial diseñado para la captura de material particulado $<10 \mu \mathrm{m}$ y una bomba de muestreo que succiona la muestra durante 24 horas en cada sitio. La concentración de $\mathrm{PM}_{10}$ se determina por tasación gravimétrica. Además, se toma en cuenta para cada determinación, el flujo de la bomba, tiempo de muestreo, presión y temperatura.

La medición del dióxido de nitrógeno se hace mediante un método de difusión pasiva basado en el Método de Palmer, que utiliza un medio absorbente donde se realiza la adsorción química del contaminante por un período de treinta días. Los análisis se realizan posteriormente por espectrofotometría a una longitud de onda de $540 \mathrm{~nm}$.

Para determinar la concentración de ozono se realiza la adsorción química del contaminante por un período de ocho días. Posteriormente se realizan los análisis por espectrofotometría, a una longitud de onda de $442 \mathrm{~nm}$.

La medición de polvos se realiza mediante la utilización del Método de Bergerhoff. En un recipiente plástico se precipita el polvo por espacio de treinta días. La concentración se determina en el laboratorio mediante separación por filtración de polvos solubles e insolubles, y determinación de cada fracción por secado al horno y tasación gravimétrica.

La medición de $\mathrm{CO}$ se realiza mediante la absorción de los contaminantes en carbón activado. Después, el carbón activado es liberado con disulfuro de carbono. Se realiza el análisis en un cromatógrafo de gases equipado con un detector de ionización de llama (FID).

La medición de plomo atmosférico se realiza utilizando un filtro de jeringa de teflón (PTFE), muestreador especial diseñado tanto para la captura de material 
particulado $<10 \mu \mathrm{m}$ como de Plomo Atmosférico, y una bomba de muestreo, la cual succiona la muestra durante 24 horas en cada sitio. La concentración se determina por digestión de la muestra y análisis posterior en un espectrofotómetro de absorción atómica equipado con horno de grafito.

El comportamiento del dióxido de nitrógeno $\left(\mathrm{NO}_{2}\right)$ no presenta grandes variaciones durante los años 1996 y 2001. El promedio durante los seis años fue de $32.66 \mu \mathrm{g} / \mathrm{m}^{3}$. Durante todo el período de estudio solamente en el año 1996 superó el valor guía que para este parámetro establece la OMS, que es de $40 \mu \mathrm{g} / \mathrm{m}^{3}$, basado en datos de UNI-CIEMA (2001) (ver ilustración 2).

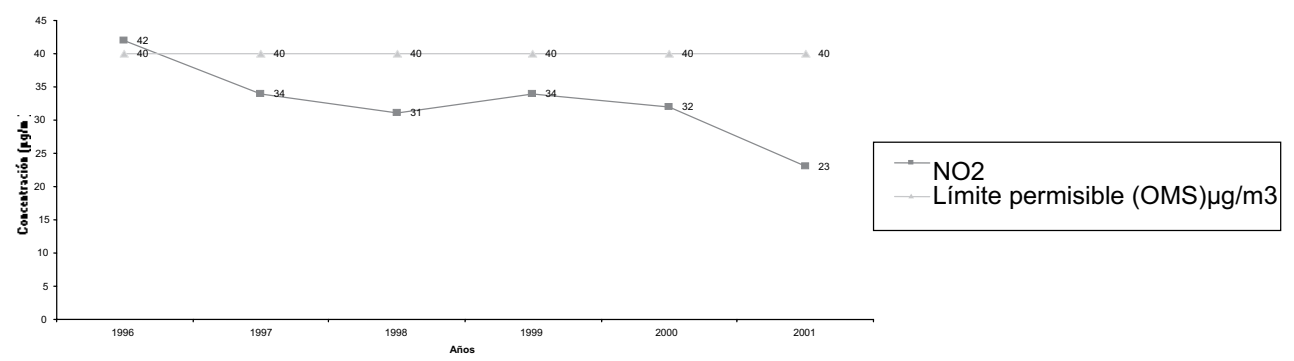

Ilustración 2. Dióxido de nitrógeno

Fuente: Autores en base a datos de contaminación del aire UNI-CIEMA (2001).

Las partículas menores a 10 micrómetros en el periodo de monitoreo de la calidad del aire están por encima de la norma establecida por la OMS, con un promedio de $64 \mu \mathrm{g} / \mathrm{m}^{3}$ con valores mínimos y máximos en los años 1999-2001 de 52 $\mu \mathrm{g} / \mathrm{m}^{3}$ y $70 \mu \mathrm{g} / \mathrm{m}^{3}$ respectivamente (ver ilustración 3 ).

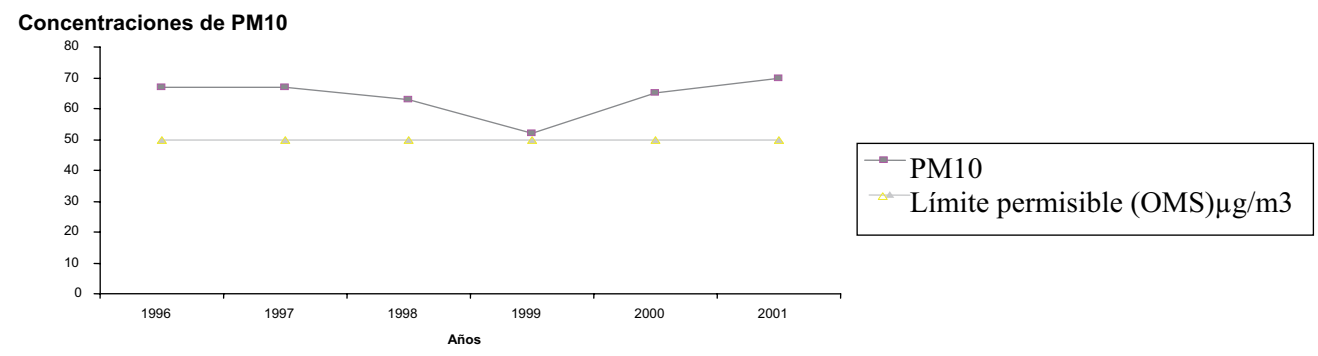

Ilustración 3. Partículas Menores de 10 micrómetros (PM10)

Fuente: Autores en base a datos de contaminación del aire, UNI-CIEMA (2001).

En el año 2001 se presentaron las menores concentraciones de ozono (O3) $23 \mu \mathrm{g} / \mathrm{m}^{3}$, alcanzado su valor máximo de $119 \mu \mathrm{g} / \mathrm{m}^{3}$ en 1999 . El valor promedio durante el monitoreo fue de $69 \mu \mathrm{g} / \mathrm{m}^{3}$, superando el valor guía establecido por la OMS de $60 \mu \mathrm{g} / \mathrm{m}^{3}$. Es importante mencionar que en los años 2000 y 2001 hubo una reducción en los niveles de emisiones dentro de los límites permitidos (ver ilustración 4). 


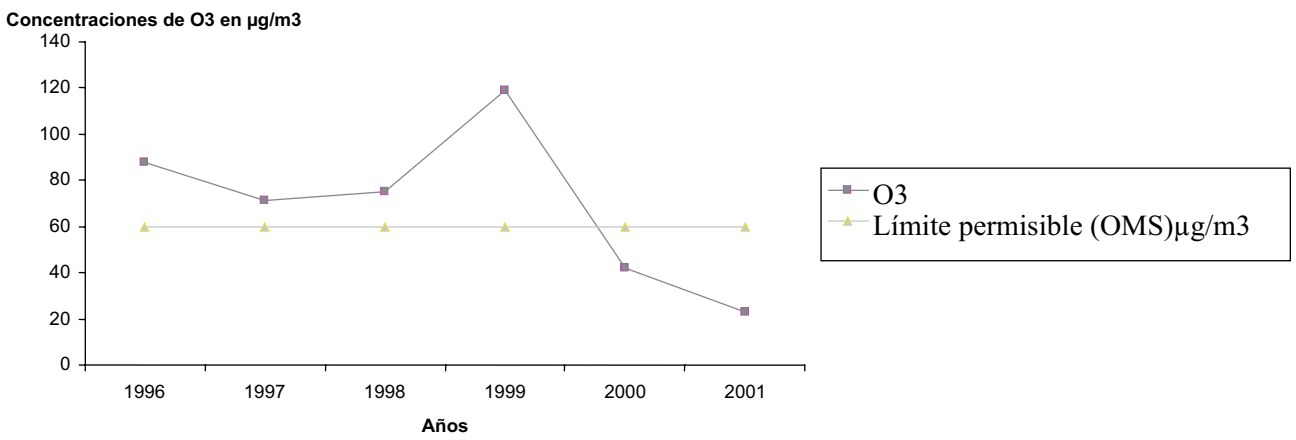

Ilustración 4. Ozono (O3)

Fuente: Autores en base a datos de contaminación del aire, UNI-CIEMA (2001).

Las partículas totales suspendidas (PTS) superaron durante todos los meses del año 2000 el valor guía que para este contaminante que estableció la OMS en $1993\left(75 \mu \mathrm{g} / \mathrm{m}^{3}\right)$.

Las mayores concentraciones se alcanzaron en 1998. Las altas concentraciones observables durante el monitoreo se dieron en la estación seca y tienen su explicación en la erosión eólica, las quemas de bosques, las tierras cultivables y el tráfico vehicular. Durante la estación lluviosa se observa un descenso en la concentración de partículas debido al efecto de lavado atmosférico que ejerce la lluvia y a la retención en la tierra de millones de partículas por efecto de la saturación del terreno a causa de las lluvias. Al establecer comparaciones entre las estaciones de monitoreo donde esta actividad se efectuó de manera periódica, la mayor concentración se alcanzó en la UNI (416 $\left.\mu \mathrm{g} / \mathrm{m}^{3}\right)$, zona de alto tráfico vehicular, y la menor en Linda Vista $\left(195 \mu \mathrm{g} / \mathrm{m}^{3}\right)$, zona residencial ubicada al noroeste de la ciudad de Managua. El promedio anual es de $285 \mu \mathrm{g} / \mathrm{m}^{3}$ (ver ilustración 5).

Concentración de PTS en $\mu \mathrm{g} / \mathrm{m} 3$
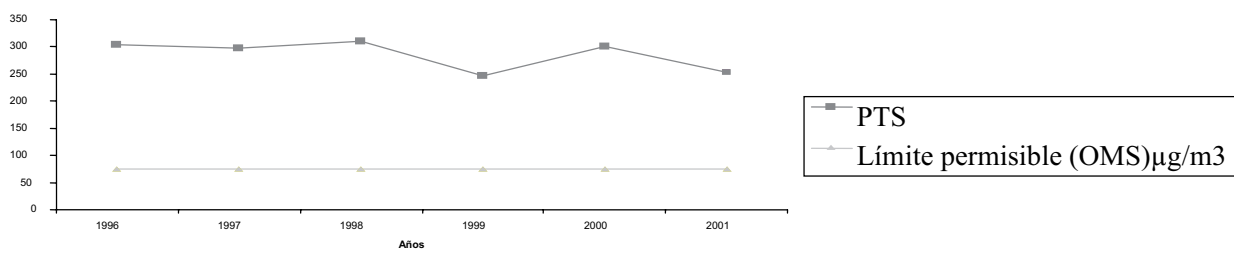

Ilustración 5. Partículas totales suspendidas (PTS)

Fuente: Autores en base a datos de contaminación del aire, UNI-CIEMA (2001).

El cuadro 1 muestra las principales estadísticas descriptivas de los datos de contaminación y de la tasa de morbilidad por diez mil habitantes en la ciudad de Managua. Estos indican que en Managua existe una tasa de morbilidad por diez mil habitantes (TMB) de 361 en promedio en los periodos 1996 y 2001. La contaminación del aire por partículas menores a 10 micrómetros $\left(\mathrm{PM}_{10}\right)$ y las partículas totales suspendidas (PTS) superaron los valores guías establecidos por la OMS. El ozono (O3) solamente en el periodo 1996-1999 superó la norma establecida. En base a las 
estadísticas se puede decir que la morbilidad o enfermedades respiratorias agudas son causadas en parte por la contaminación del aire especialmente por PTS y PM10.

Cuadro 1. Estadística descriptiva de la tasa de morbilidad por diez mil habitantes y la contaminación del aire en Managua (1996-2001)

\begin{tabular}{|l|c|c|c|c|c|}
\hline \multicolumn{1}{|c|}{ Variables } & $\begin{array}{c}\text { No. de } \\
\text { observaciones }\end{array}$ & Promedio & $\begin{array}{c}\text { Desviación } \\
\text { Estándar }\end{array}$ & $\begin{array}{c}\text { Valor } \\
\text { mínimo }\end{array}$ & $\begin{array}{c}\text { Valor } \\
\text { máximo }\end{array}$ \\
\hline TBM & 6 & 361 & 40.71 & 307 & 433 \\
\hline $\mathrm{NO}_{2}$ & 6 & 32.66 & 6.12 & 23 & 42 \\
\hline $\mathrm{PM}_{10}$ & 6 & 64.00 & 6.32 & 52 & 70 \\
\hline O3 & 6 & 69.66 & 33.89 & 23 & 119 \\
\hline PTS & 6 & 285.00 & 28 & 246 & 310 \\
\hline Tráfico automotor & 5 & 102400 & 20935.62 & 75000 & 127000 \\
\hline
\end{tabular}

Fuente: Autores en base a datos de contaminación del aire, UNI-CIEMA (2001) y MINSA (2005)

La matriz de correlación parcial para la tasa de morbilidad por diez mil habitantes (TBM) en correspondencia principalmente con las partículas totales suspendida (PTS) presentada en el cuadro 2 muestra una relación directa entre TBM y PTS, ya que se correlacionan positivamente un $30 \%$, el ozono $8 \%$ y las PM10 con un $4 \%$. Esto indica que el mayor impacto sobre la morbilidad lo ocasionan los PTS y el O3 (ver cuadro 2).

Cuadro 2. Matriz de correlación

\begin{tabular}{|l|r|}
\hline Variables & TBM \\
\hline PM10 & 0.04 \\
\hline O3 & 0.08 \\
\hline PTS & 0.3 \\
\hline
\end{tabular}

Fuente: cálculo de los autores

En Estados Unidos las principales fuentes de contaminación del aire son el transporte terrestre y los procesos industriales con porcentajes de $43 \%$ y $25 \%$ (ver ilustración 6).
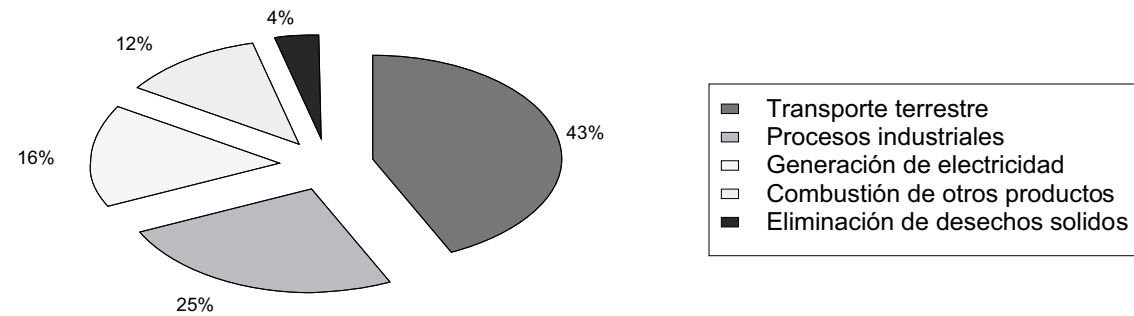

Ilustración 6. Principales fuentes de problemas ambientales en \%

Fuente: Parkin, 2001 
En Managua el transporte público es obsoleto, por lo que hemos de esperar que sea el de mayor contribución a la contaminación. Además, se tiene una industria de poco desarrollo tecnológico. Otro problema que puede incrementar la contaminación del aire es el alto consumo de leña para cocinar que a nivel nacional es de 59\% y en la zona rural de 97\%. En Managua, de acuerdo a la Encuesta de Medición del Nivel de Vida (INIDE, 2005), el 27\% de las viviendas cocinan con leña (ver ilustración 7).

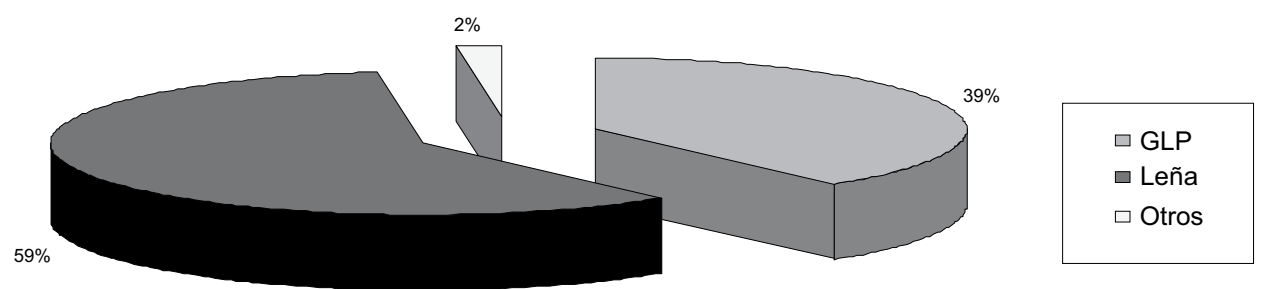

Ilustración 7. \% del consumo de combustibles para cocinar en los hogares en Nicaragua

Fuente: Autores en base a la Encuesta Nacional de Leña 2005

\section{Revisión literaria}

Gerking y Stanley (1986) plantean que los individuos producen un estatus de salud y están dispuestos a pagar por una reducción en los niveles de contaminación del aire. El modelo aplicado se basa en los trabajos realizados por Grossman (1972), Cropper (1981), Rosenzmeig and Schcults (1982) (citados por Gerking \& Stanley, 1986). Argumentan que los individuos derivan utilidad del consumo de dos clases de bienes: su propio stock de capital de salud y los bienes que les producen directamente satisfacción, pero no afectan la salud. Los autores analizaron en la ciudad de St. Louis el comportamiento de 2,197 personas para los años (1977-1980), en las que utilizaron información sobre los gastos médicos, salario, variables socioeconómicas y calidad del aire. Encontraron que las personas estaban dispuestas a pagar 25 dólares por una reducción del 30\% en la concentración de ozono.

Ostro (1998) estima el impacto de la contaminación del aire en la salud. Para esto es necesario resolver: las relaciones entre morbilidad y contaminación, la población susceptible y los cambios en los niveles de contaminación. Para estimar los efectos de la contaminación del aire en la salud, propone una metodología basada en estudios de series cronológicas que relacione cambios diarios en la contaminación atmosférica con la incidencia diaria de un efecto sobre la salud. En el caso de Santiago de Chile, a partir de datos locales disponibles, al aplicar esta metodología se obtiene un cambio porcentual en mortalidad del $1 \%$ asociado a cambios de 10 $\mu \mathrm{g} / \mathrm{m}^{3}$ en concentraciones de $\mathrm{PM}_{10}$. En este estudio se encontró que los efectos sobre la mortalidad tienden a dominar los costos económicos de la contaminación atmosférica.

Castillo, Torres y Fernández (2001) resumen los efectos de los niveles de contaminación reportados en México y encuentran que un incremento en 10 
unidades de $\mathrm{PM}_{10} \mu \mathrm{g} / \mathrm{m}^{3}$ produce un efecto agudo en la mortalidad de $0.96 \%$, en hospitalizaciones de $1.39 \%$, en visitas a salas de urgencia de $3.11 \%$ y en síntomas respiratorios de $7.72 \%$. Estos resultados muestran evidencia del gran impacto que las concentraciones de contaminantes del aire causan a la salud.

Ibáñez y McConnell (2001) valoran la morbilidad por los efectos de contaminación del aire en Bogotá, Colombia. Los datos utilizados en este estudio se basan principalmente en dos fuentes de información: datos de los niveles de contaminación del aire e información de morbilidad de las admisiones diarias de los hospitales. El documento examina la relación entre la contaminación del aire y las admisiones diarias por infecciones respiratorias agudas a los hospitales. Ésta resulta ser positiva. En este estudio es importante mencionar que se encuentra que a las personas de mayor edad les afecta más las partículas suspendidas y a los jóvenes el dióxido de nitrógeno.

Germany (2003) recomienda que el estudio de la contaminación del aire sea un requisito para el desarrollo de políticas efectivas que contribuyan a reducir el impacto al ambiente de los niveles altos de concentraciones de contaminantes. Presenta evidencia de cómo las $\mathrm{PM}_{10}$ han incrementado la mortalidad en Europa y cómo el ozono produce efectos a corto plazo sobre mortalidad y morbilidad respiratoria. Argumenta que es necesario establecer políticas que ayuden a mejorar la calidad del aire.

Brugman (2004) realizó un estudio con el objetivo de elaborar una propuesta de política para el control de la contaminación atmosférica urbana en Colombia. Estimó para varias ciudades una proyección de demanda por combustibles para los años 2004-2020 a partir de las proyecciones de demanda y mediante la aplicación de factores de emisión de contaminantes asociados a cada uso de los combustibles $\left(\mathrm{PM}_{10}, \mathrm{NO}_{\mathrm{x}}\right.$ y $\left.\mathrm{CO}\right)$. En el caso de la proyección de las emisiones por fuentes móviles, sobresale el aporte del material particulado menor a diez micras $\left(\mathrm{PM}_{10}\right)$, puesto que este contaminante genera graves dificultades sobre la salud de la población, en especial de la población menor de cinco años. Él propone un ajuste de precio en la gasolina usando la regulación del método paridad de las importaciones, lo que permitiría una disminución en la contaminación al inicio de 2.3\%, hasta llegar al $7.3 \%$ en 2020.

\section{Resultados}

La literatura descrita anteriormente sirvió como un marco para determinar los alcances que debía tener el ensayo sobre los efectos esperados de reactivar el proyecto de contaminación del aire, los estudios sobre los costos de enfermarse por infecciones respiratorias agudas y las recomendaciones de políticas para reducir la contaminación del aire.

Para el análisis de la morbilidad y el impacto en la salud humana, consideramos las enfermedades relacionadas con la contaminación atmosférica como: infecciones respiratorias agudas, asma y bronquitis. Estudios realizados en México y Santiago de Chile muestran que este conjunto de enfermedades respiratorias se relacionan de forma directa con la contaminación del aire, en particular con la concentración de 
partículas suspendidas emitidas primordialmente por fuentes móviles y fijas.

En nuestro trabajo tomamos como población de referencia a los menores de 15 años y a los mayores de 50 años, lo que representa el 94\% del total de casos reportados en el periodo 1996 a 2001.

Esta restricción se plantea bajo el supuesto de que la población infantil y los de la tercera edad son los que presentan mayores afectaciones (ver ilustración 8).

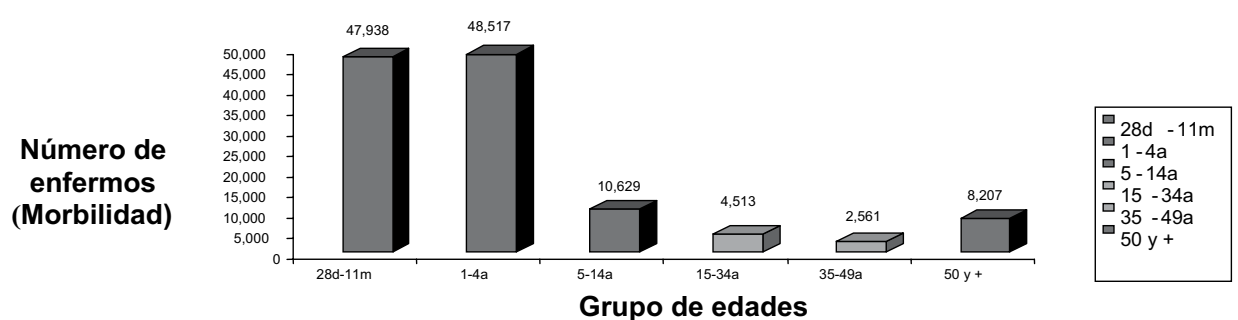

Ilustración 8. Morbilidad en los años (1996 a 2001)

Fuente: Autores en base a los datos del Ministerio de Salud (MINSA, 2005)

De acuerdo a la EMNV año 2005 (INIDE, 2005), los problemas respiratorios como tos y resfrío representan el $28.62 \%$ de las enfermedades que tuvieron las personas un mes antes a la encuesta. Los casos de diarrea para las personas de 6 años o más solamente significan el $0.62 \%$. Lo importante de la ilustración número 9 es resaltar que el $61.32 \%$ de las personas que se enfermaron por problemas respiratorios son pobres. El gasto en salud tiene un gran impacto negativo en sus ingresos ya que demandarán medicamentos principalmente en actividades curativas.
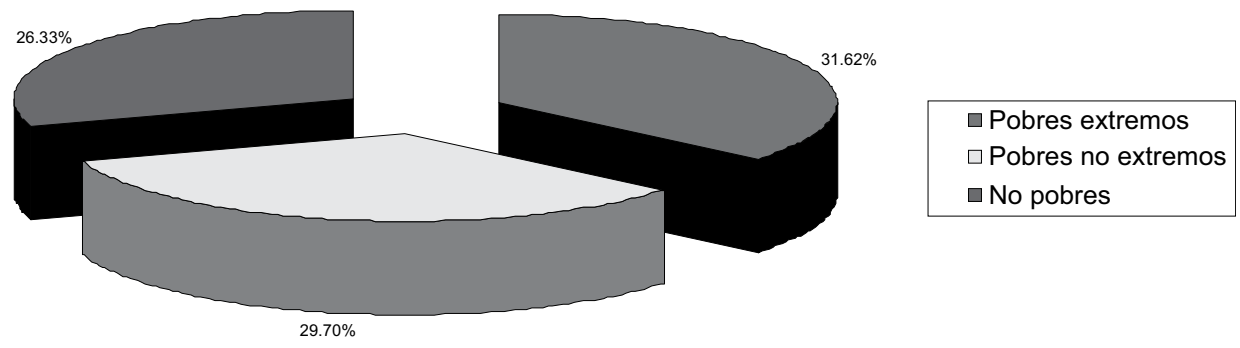

Ilustración 9. Enfermedades respiratorias por niveles de pobreza

Fuente: Autores en base a los datos de EMNV 2005 (INIDE, 2005)

Los lugares en donde se consulta por enfermedad respiratoria son los centros de salud y los hospitales públicos con $67 \%$ (ver ilustración 10). Estos son los sitios en donde debe de existir un stock de medicamentos para satisfacer la demanda de casos por morbilidad, especialmente ocasionados por la contaminación del aire. En el estudio se hizo una entrevista al medico de turno en el Centro de Salud Sócrates Flores y descubrimos que básicamente recetan acetaminofen de 500 miligramos por 5 días cada seis horas. Esto origina un gasto en medicamentos de 20 córdobas por persona en los centros de salud en Managua. Es importante mencionar que lo 
recomendado por el doctor son 5 días de subsidio, lo que origina pérdidas en la productividad de actividades económicas de las personas, provocando afectaciones en los sitos de trabajo.

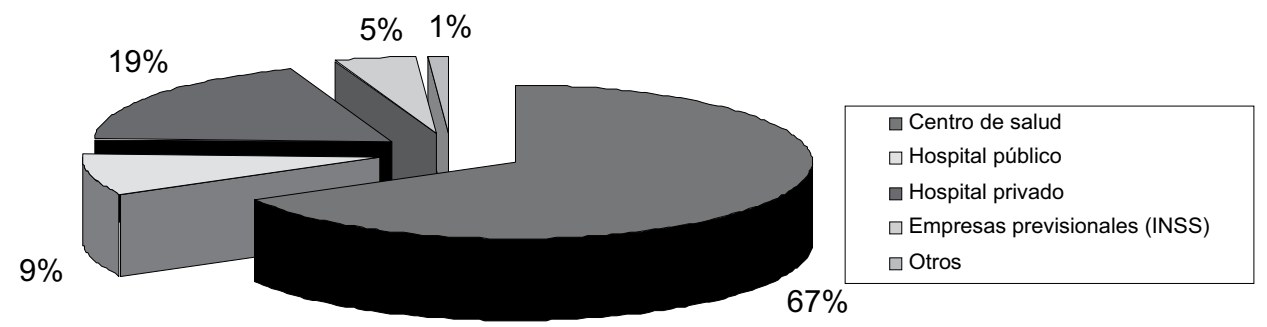

Ilustración 10. Lugar de consulta por tos y resfrío

Fuente: Autores en base a los datos de EMNV 2005 (INIDE, 2005)

En el cuadro 3 se presentan las variables que influyen cuando una persona se enferma con tos y resfrío, y asiste a un centro de salud.

Cuadro 3. Estadística de las principales variables

\begin{tabular}{|l|c|}
\hline Variables & Media \\
\hline Tiempo en minutos de demora de la casa a la consulta (ida y regreso) & 30 min \\
\hline Costo en córdobas de transporte de ida y regreso de la consulta & 5 C $\$$ \\
\hline Tiempo en minutos de espera para ser atendido en la consulta & 40 min \\
\hline Tiempo en minutos en la consulta & $10 \mathrm{~min}$ \\
\hline Gasto en córdobas en medicamentos para tratarse la tos y el resfrío & $73 \mathrm{C} \$$ \\
\hline
\end{tabular}

Fuente: Autores en base a los datos de EMNV 2005 (INIDE, 2005)

En Nicaragua, tomando como base los indicadores del Banco Central, el salario mínimo dado a los pensionados es de 2,285 córdobas mensuales y el salario promedio de los asegurados en el año 2008 fue de 5,057.30 córdobas por mes.

En la investigación utilizaremos el tiempo como un costo de oportunidad que una persona realiza por ir a pasar consulta. Existen estudios en los que se ha demostrado que el costo de oportunidad en el tiempo por desplazamiento tiene un valor sobre el salario comprendido entre el 25\% y 50\% (Smith \& Kaoru, 1990). 
Cuadro 4. Forma de estimación del costo de oportunidad

\begin{tabular}{|c|c|}
\hline Salario diario (INSS) & Salario diario mínimo \\
\hline $\mathrm{CO}^{\mathrm{DIA}}=\frac{\operatorname{Tg}_{\min }^{\mathrm{dia}}[\operatorname{Salario}(1-0.25)]}{\mathrm{Tt}_{\min }^{\mathrm{dia}}}$ & $\mathrm{CO}^{\mathrm{DIA}}=\frac{\operatorname{Tg}_{\min }^{\mathrm{dia}}[\text { Salario }(1-0.25)]}{\mathrm{T}_{\mathrm{min}}^{\mathrm{dia}}}$ \\
\hline $\mathrm{Tg}_{\min }^{\text {día }}$ Tiempo gastado en el viaje min/día. & $\operatorname{Tg}_{\min }^{\text {día }}$ Tiempo gastado en el viaje min/día. \\
\hline $\mathrm{Tt}_{\min }^{\mathrm{dia}}$ Tiempo total de trabajo min/ día. & $\mathrm{Tt}_{\min }^{\mathrm{dia}}$ Tiempo total de trabajo min/ día. \\
\hline $\mathrm{Tt}_{\mathrm{min}}^{\mathrm{dia}}=\frac{60 \mathrm{~min} * 8 \mathrm{~h}}{1 \mathrm{~h}}=480 \mathrm{~min} /$ día & $\mathrm{Tt}_{\min }^{\mathrm{dia}}=\frac{60 \mathrm{~min}^{*} 8 \mathrm{~h}}{1 \mathrm{~h}}=480 \mathrm{~min} /$ día \\
\hline
\end{tabular}

Fuente: Autores en base a Smith \& Kaoru (1990)

En el tiempo gastado en el viaje min/día se incluyen el tiempo en minutos de demora de la casa a la consulta, el tiempo en minutos de esperar para ser atendido por el doctor y el tiempo en minutos en la consulta. Esto da una sumatoria en base al cuadro 3 de 80 minutos equivalente a 1 hora con 20 minutos.

Cuadro 5. Resultado de la estimación del costo de oportunidad

\begin{tabular}{|l|l|}
\hline \multicolumn{1}{|c|}{ Salario diario (INSS) } & \multicolumn{1}{c|}{$\begin{array}{c}\text { Salario diario mínimo (INSS) } \\
\text { Pensionados }\end{array}$} \\
\hline$C O^{D I A}=\frac{80[169(1-0.25)]}{480}=21 C \$$ & $C O^{D I A}=\frac{80[76(1-0.25)]}{480}=3 C \$$ \\
$T g_{\text {min }}^{\text {dia }}=80 \mathrm{~min}$ & $T g_{\text {min }}^{\text {dia }}=80 \mathrm{~min}$ \\
$T t_{\text {min }}^{\text {dia }}=\frac{60 \mathrm{~min} * 8 h}{1 \mathrm{~h}}=480 \mathrm{~min} /$ día & $T t_{\min }^{\text {dia }}=\frac{60 \mathrm{~min} * 8 \mathrm{~h}}{1 \mathrm{~h}}=480 \mathrm{~min} /$ día \\
Salario $=5,057.30 \mathrm{C} \$ / \mathrm{Mes}$ & Salario $=2,285 \mathrm{C} \$ / \mathrm{Mes}$ \\
Salario $=169 \mathrm{C} \$ /$ día & Salario $=76 \mathrm{C} \$$ día \\
\hline
\end{tabular}

Fuente: Autores en base a Smith \& Kaoru (1990)

Para determinar el impacto de la contaminación del aire sobre la tasa de morbilidad por diez mil habitantes (TBM) calculamos las elasticidades, que es una medida del cambio porcentual de una variable (TBM) provocada por el cambio de uno por ciento de otra variable, en nuestro caso contaminación del aire por: PM10, PTS y O3. 
Cuadro 6. Elasticidades

\begin{tabular}{|l|r|}
\hline Variables & Elasticidades \\
\hline PM10 & 15 \\
\hline O3 & 5.34 \\
\hline PTS & 7.07 \\
\hline Trafico automotor & 7.45 \\
\hline
\end{tabular}

Fuente: Cálculo de los autores

Los resultados del cuadro 3 muestran que un incremento en $1 \%$ de las PM10 provoca un aumento del $15 \%$ en la tasa de morbilidad, un incremento en las PTS provoca un aumento del 7.07\%, un incremento en el ozono (O3) del 5.34\% y el crecimiento del $1 \%$ del parque automotor provoca un aumento en la tasa de morbilidad de $7.45 \%$.

Se puede establecer la relación entre contaminación del aire y crecimiento económico. Para ello utilizamos un análisis de correlación parcial. El cuadro 7 muestra los resultados. En él se observa una relación directa entre la TBM y las tasas de crecimiento del PIB de $21 \%$, con la tasa de crecimiento de la demanda interna en un $25 \%$, con la tasa de crecimiento de la inversión privada en un $24 \%$, con la tasa de crecimiento de las importaciones en un $23 \%$ y con la tasa de crecimiento de las exportaciones negativamente en un $8 \%$, esto nos indica que el mayor impacto sobre la morbilidad lo ocasiona el crecimiento de la demanda interna, la inversión privada y las importaciones.

Cuadro 7. Matriz de correlación

\begin{tabular}{|l|r|}
\hline Variables & TMB \\
\hline Tasa de crecimiento PIB & 0.21 \\
\hline Tasa de crecimiento del consumo & 0.25 \\
\hline Tasa de crecimiento de la inversión privada & 0.24 \\
\hline Tasa de crecimiento de la importaciones & 0.23 \\
\hline Tasa de crecimiento de las exportaciones & -0.08 \\
\hline
\end{tabular}

Fuente: Cálculo de los autores en base a datos indicadores económicos del Banco Central de Nicaragua (2010) .

La relación existente entre el consumo y la tasa de morbilidad puede ser atribuida al alto consumo de leña para cocinar ${ }^{1}$, mientras que la relación con la inversión privada y a la vez con las importaciones puede ser atribuida a la alta tasa de importación de automotores en el periodo estudiado, ya que dicha tasa haciende a $14.16 \%$ promedio anual a nivel nacional. Por otro lado, la relación negativa con

1 Como es conocido, en países con niveles bajos de ingresos la mayoría del consumo es de subsistencia, es decir, para alimentación. Dicha regla intuición es conocida en la teoría económica como la ley de Engel. 
las exportaciones no es más que una manifestación de que los productos que se exportan son eminentemente agropecuarios y por tanto, de poca influencia en la contaminación atmosférica.

En 1996 nace La Ley General del Medio Ambiente y los Recursos Naturales de Nicaragua (ley No. 217) (Asamblea Nacional, 1996). En dicha ley se establece que el Estado tiene el deber de garantizar la prevención de los factores ambientales adversos que afecten la salud y la calidad de vida de la población ${ }^{2}$ (arto. 109), que el encargado de regular la política ambiental del país será el Ministerio del Ambiente y Recursos Naturales (arto. 8), que se orientará el monitoreo y el control de las fuentes fijas y móviles (arto. 111) y que se creará un Fondo nacional del ambiente para desarrollar y financiar programas y proyectos de protección, conservación y restauración del medioambiente y desarrollo sostenible financiado a través de fondos obtenidos de otorgamiento de licencias ambientales, multas y decomisos por infracciones a esta ley y por donaciones nacionales e internacionales para este fin (artos. 49-51).

En la ley 217 también se manda a emitir estándares de gases contaminantes (artos 11 y 112) (Asamblea Nacional, 1996). En respuesta a esto surge el decreto 32 97 Reglamento general para el control de emisiones de vehículos automotores posteriormente reformada por el decreto 22-98 (Asamblea Nacional, 1998). En dichas leyes se establece que los vehículos con motor a gasolina que circulasen antes del 1 de enero de 1999 no deben emitir monóxido de carbono (CO) en cantidades superiores al $4.5 \%$ del volumen total de los gases, ni hidrocarburos (HC) en cantidades superiores a 600 ppm (partes por millón), ni dióxido de carbono (CO2) en cantidades inferiores al 10.5\% del volumen total de los gases (arto 20-21). Además, que los vehículos con motor a gasolina que ingresen al país de manera permanente después del 1 de enero de 1999, no deben emitir monóxido de carbono (CO) en cantidades superiores a $0.5 \%$ del volumen total de los gases, ni hidrocarburos (HC) en cantidades superiores a 125 ppm, ni bióxido de carbono en cantidades menores al 12\% del volumen total de los gases.

Por otro lado, los vehículos con motor a diesel que hayan ingresado al país de manera permanente antes del 1 de enero de 1999, con un peso menor o igual a 3.5 toneladas, no deberán emitir humos y partículas cuya opacidad exceda el $70 \%$, excepto aquellos vehículos que funcionen con motores diesel turboalimentados, cuyo límite de emisión no podrá superar el $80 \%$ de opacidad. Por otro lado, los vehículos con un peso mayor a 3.5 toneladas no deberán emitir humos y partículas cuya opacidad exceda el $80 \%$ (arto 22). Además, que los vehículos con motor a diesel con peso igual o menor a 3.5 toneladas que hayan ingresado al país de manera permanente después del 1 de enero de 1999, no deben emitir humos y partículas cuya opacidad exceda el 60\%, excepto los vehículos con motores diesel turboalimentados, cuyo límite de emisión no podrá exceder el 70\% de opacidad. Por otro lado, que los vehículos con peso mayor a 3.5 toneladas no deberán emitir humo o partículas cuya opacidad exceda el $70 \%$.

\footnotetext{
2 Este artículo está contenido en el artículo 60 de la constitución política, el cual afirma que "los nicaragüenses tienen el derecho de habitar en un ambiente saludable y que es obligación del estado la preservación, conservación y rescate del medio ambiente y de los recursos naturales".
} 
Los estándares de emisión establecidos en estas leyes se basan principalmente en la revisión de la literatura internacional. Los beneficios, eficacia y eficiencia de esas normas no se han evaluado rigurosamente, dado que los esfuerzos gubernamentales para evaluar el impacto del cumplimiento de dicha normativa en la calidad del aire han sido muy restringidos por la falta de información estadística necesaria para una evaluación cuantitativa.

Las leyes aprobadas en los noventa deben ser modificadas a la luz de nueva información acerca de los efectos de la contaminación atmosférica en la salud. Dicha información en Nicaragua es escasa o inexistente a causa de la falta de información estadística necesaria que brinde evidencia empírica de los efectos de la contaminación en la salud humana. Dicha evidencia debe formar parte de un proceso de reelaboración de la normativa actual y del diseño de políticas gubernamentales sobre la prevención y el control de la contaminación del aire.

El punto de partida para la solución del problema actual de la regulación ambiental es crear un registro permanente y frecuente en las estadísticas relacionadas con la contaminación ambiental, ya que desde el año 2001 no existe un monitoreo de la calidad del aire en Managua y menos en otros departamentos. Además, es muy importante involucrar a los centros de salud para poder determinar con exactitud el porcentaje de pacientes con infecciones respiratorias agudas causadas por la contaminación del aire.

De acuerdo a la UNI, volver a activar el programa de monitoreo de la calidad del aire por medio del Centro de Investigaciones en Medio Ambiente (CIEMA) en los mismos puntos y contaminantes, cuesta 30,000 dólares anuales y genera 10 empleos nuevos.

Cuadro 8. Subsidio al transporte en Managua

\begin{tabular}{|r|r|}
\hline \multicolumn{2}{|l|}{$\begin{array}{l}\text { Transferencias corrientes del gobierno en millones de córdobas } \\
\text { anuales a cooperativas de transporte urbano colectivo en Managua }\end{array}$} \\
\hline Años & C \$/anuales \\
\hline 2007 & 132.574 \\
\hline 2008 & 141.400 \\
\hline 2009 & 120.000 \\
\hline 2010 & 120.000 \\
\hline
\end{tabular}

Fuente: Indicadores económicos del Banco Central de Nicaragua (2010).

Se necesita activar el programa de monitoreo del aire para poder aplicar la regulación ambiental usando la ley 217 (Asamblea Nacional, 1996). Por tanto, es necesario obtener el financiamiento y esto se puede lograr haciendo que el contaminador pague un impuesto equivalente al valor del monitoreo, que tomando como base la ilustración 6 sería el transporte terrestre en Managua, que evidentemente por el estado deteriorado en el que se encuentran los buses, debe ser el principal causante de los estándares elevados de PM10 y PTS encontrados durante el monitoreo de la calidad del aire. 
De la transferencia que hace el gobierno por subsidio de combustible (cuadro 8) se les debe de descontar el daño ambiental por 700 mil córdobas anuales. Este dinero debe ser usado para el monitoreo de la calidad del aire. El sector transporte estaría recibiendo en el año 2010 un monto de 119 millones con 300 mil córdobas, no afectando la tarifa de los $2.50 \mathrm{C} \$$.

El cuadro 9 muestra el poco impacto que esta medida tendría en la tarifa, ya que prácticamente estarían pagando 1.60 córdobas por día, una cifra menor al precio que paga un pasajero por usar el bus (2.50 C\$/viaje) en un viaje. Es importante mencionar que este cálculo se realizó tomando como referencia una fuente anónima que menciona que en Managua existen 1,200 unidades de transporte colectivo.

Cuadro 9. Impacto del daño ambiental en la tarifa en el transporte

\begin{tabular}{|l|r|r|}
\hline Concepto & $\begin{array}{l}\text { Sin tomar en cuenta el } \\
\text { daño ambiental } \\
\text { C\$/Año }\end{array}$ & $\begin{array}{l}\text { Tomando en cuenta el } \\
\text { daño ambiental } \\
\text { C } \$ \text { /Año }\end{array}$ \\
\hline Subsidio al transporte & $120,000,000.00$ & $119,300,000.00$ \\
\hline Monto a recibir por unidad en el año & $100,000.00$ & $99,416.67$ \\
\hline Monto a recibir por unidad en el mes & $8,333.33$ & $8,284.72$ \\
\hline
\end{tabular}

Fuente: Autores en base a indicadores del Banco Central de Nicaragua (2010) y fuente anónima.

La otra alternativa es que sacrifiquen los $1.60 \mathrm{C} \$$ de las ganancia por día en un bus para que no se afecte la tarifa de los $2.50 \mathrm{C} \$$ y sea éste un aporte de las cooperativas a la sociedad por el daño causado.

Los $1.60 \mathrm{C} \$$ por día en una unidad de transporte es una cifra mucho menor de lo que pierde una persona como costo de oportunidad por viaje (ver cuadro 5) a la consulta; que para las personas con salario mínimo es de $3 \mathrm{C} \$$.

Existe un gasto en salud ocasionado por la contaminación del aire, especialmente por $\mathrm{PM}_{10}$. Si tomamos como referencia solamente los $20 \mathrm{C} \$$ por persona en actividades curativas (acetaminofen) y los 30 mil casos en promedio reportados por el SILAIS, que de acuerdo a la matriz de correlación del cuadro 2 las $\mathrm{PM}_{10}$ y las PTS causan el 34\% de las enfermedades respiratorias agudas, se debe de proveer a los SILAIS de un stock disponible en medicamentos tanto para actividades curativas como preventivas y pagos a los doctores por consulta.

Otra alternativa más es gravar un impuesto al consumo de diesel, ya que representa un precio menor y mayores consumos (ver ilustraciones 11 y 12) de los tipos de combustibles usados en el país. 


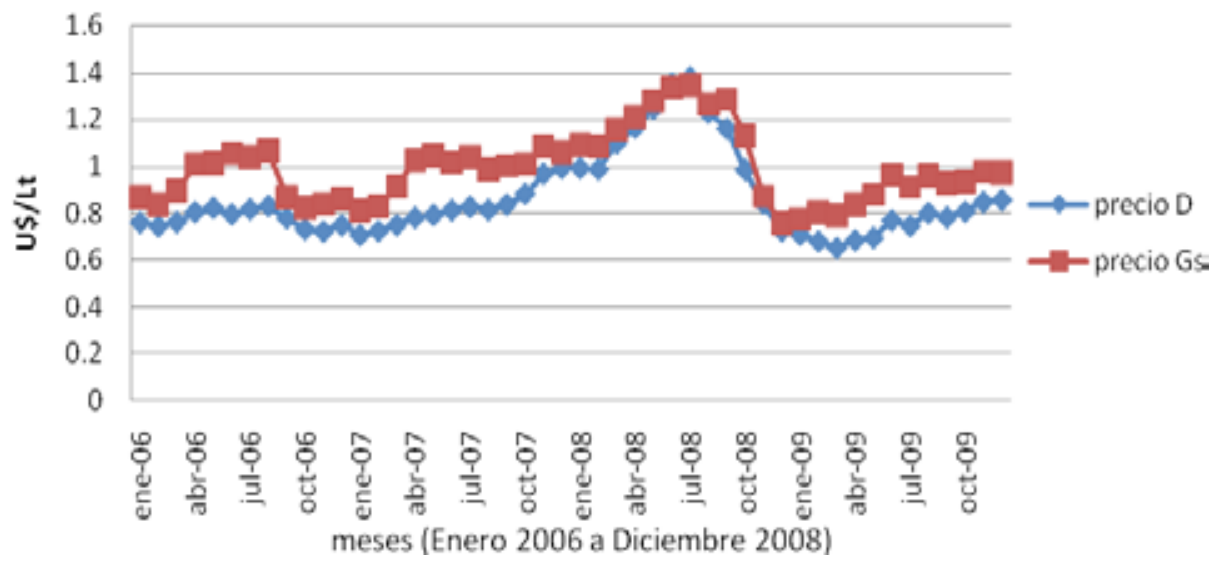

Ilustración 11. Precios del Diesel (D) y la Gasolina Súper (Gs) en (U\$/Lt)

Fuente: Autores en base a datos del INE (2006, 2007, 2008, 2009).

De acuerdo a la ilustración 11, en julio de 2008 se alcanzaron los precios máximos de $1.37 \mathrm{U} \$$ por litro para el diesel y $1.34 \mathrm{U}$ \$ por litro para la gasolina súper.

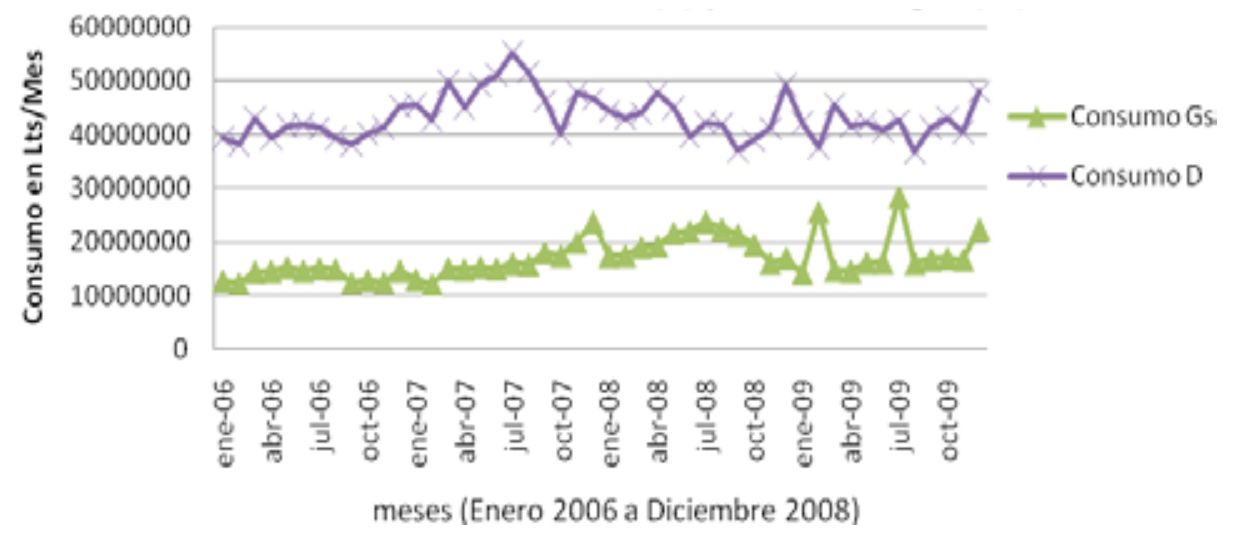

Ilustración 12. Consumo de Diesel (D) y Gasolina Súper (Gs) en litros

Fuente: Autores en base a datos del INE (2006, 2007, 2008, 2009).

Los consumos máximos de combustibles son aproximadamente de 55 millones de litros de diesel y 22 millones de litros de gasolina súper al mes. Si el gobierno grava un impuesto en uno de los 12 meses del año (el de mayor consumo de diesel) equivalente a 0.0047 centavos dólar por litro o $0.11 \mathrm{C} \$$ por litros, recaudaría para proyectos de monitoreo, reducción a la contaminación del aire y control de las emisiones, unos 200 mil dólares, dinero suficiente para la financiación del proyecto.

Es importante mencionar que la curva de demanda de diesel presentada en la ilustración 13 no tendría ningún movimiento, ya que se observa un cumplimiento de la ley de la demanda en el tramo donde el precio del diesel supera la unidad en dólares por litro. Por otro lado, cuando el precio por litro es menor a 1 dólar, se comporta como un bien Giffen, es decir, ante un aumento del precio, el consumo también aumenta. Por tanto, un impuesto en este tramo no presenta inconvenientes. 


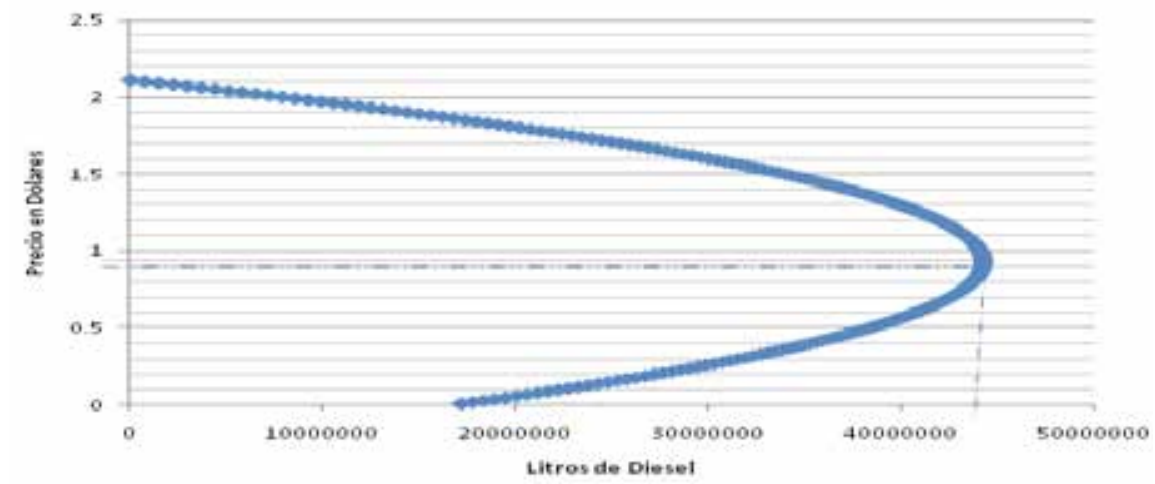

Ilustración 13. Demanda de Diesel en meses (enero 2006 a diciembre 2009) Fuente: Autores en base a datos del INE

\section{Conclusiones y recomendaciones}

Tomando como base los datos de monitoreo de la calidad del aire y los datos de morbilidad, existe una relación entre las partículas menores a 10 micrómetros, las partículas totales suspendidas (PTS), el ozono $\left(\mathrm{O}_{3}\right)$-causados por el crecimiento del tráfico vehicular y la contaminación-, y la tasa de morbilidad en Managua. El gasto en salud es de 810, 000.00 córdobas anuales, sumando los medicamentos empleados en actividades curativas, el costo del transporte por ir al centro de salud y el costo de oportunidad en el tiempo por viaje y tiempo de espera en la consulta. El programa de monitoreo de la calidad del aire cuesta 30 mil dólares en 5 puntos de monitoreo por año, sin comprar equipos. De acuerdo a información teórica, el sector que mayor incide en los niveles de emisiones es el transporte terrestre, por tanto, es el que se debe regular para internalizar el daño ocasionados a las personas -siguiendo la lógica de que el que contamina paga- y para esto se necesita una ley (actualmente existe), pero combinada con un instrumento económico (impuesto ambiental).

Las leyes de medio ambiente y recursos naturales aprobadas en los años noventa se basan en opiniones de expertos y revisión de literatura internacional. Pero los beneficios, eficiencia y eficacia de dicha norma no se han evaluado rigurosamente. Para poder hacer dicho análisis se requiere de estudios cuantitativos. Dichos estudios en Nicaragua son escasos o inexistentes debido a la falta de información estadística.

Las leyes deben tomar los análisis cuantitativos de los daños de la contaminación como una base objetiva para determinar el nivel de primacía de las diferentes políticas. Se debe dar prioridad a las políticas que ataquen los contaminantes que más daño hacen.

Los efectos de la contaminación atmosférica en la salud son poco estudiados en Nicaragua. Los efectos negativos del material particulado (PTS), partículas menores a 10 micrones (MP10) y ozono (o3) en las enfermedades respiratorias no son estudiados. Es por ello que existe una necesidad de crear un centro de estudios que analice permanentemente los efectos negativos de la contaminación atmosférica 
en la salud. Dichos estudios deben formar parte de un proceso de reelaboración de la normativa ambiental.

Es necesario aumentar la conciencia pública del problema ambiental. Para ello se requiere del auxilio de los medios de comunicación, el sistema judicial y la fiscalía general. Se debe promover la conciencia sobre la contaminación ambiental y el uso de acciones legales.

El impuesto ambiental óptimo propuesto es de 0.0047 centavos dólar por litro en el mes de mayor consumo de diesel en el año. La cantidad de dinero recaudado se usaría para aplicar la legislación actual y activar el programa de monitoreo de la calidad del aire, ya que desde el 2001 no se registran datos. Esto vuelve inviable la actual legislación pues no se tiene base para poder aplicarla. La segunda mejor solución es cobrar directamente del subsidio al transporte el daño que ocasionan a la sociedad y activar con ese monto el programa de monitoreo de calidad del aire.

El gobierno debe promover la modernización del transporte ya que ayudará a reducir los niveles de emisiones, aunque no se puede medir el impacto cuantitativamente de esta política a corto plazo por que no existe información sobre la calidad del aire.

Actualmente existen medidas -aunque no necesariamente para reducir la contaminación- como la restricción en el horario de trabajo de los taxis en Managua y la introducción de unidades nuevas de transporte en los buses rusos. Es de esperar que éstos tengan un beneficio positivo en la contaminación del aire debido al mejor funcionamiento de su sistema, aunque el gobierno no dimensionó esto como beneficio ambiental.

Existen experiencias como la de Bogotá, Colombia, con el transporte moderno implementado en el 2000 y políticas de restricción de pico y placa (es decir, un día de la semana no circula un dueño de auto en la ciudad tomando como base el número de placa). Se ha comprobado que esto ha ayudado a disminuir los niveles de contaminación y reducir el gasto en salud pública.

\section{Referencias bibliográficas}

Asamblea Nacional. (1996). Ley general de medio ambiente y recursos naturales, Ley No. 217, La Gaceta Diario Oficial, (105), 27 de marzo. Recuperado el 4 de junio de 2010, de http://legislacion.asamblea.gob.ni/Normaweb.nsf/(\$All)/1B5E FB1E58D7618A0625711600561572?OpenDocument.

Asamblea Nacional. (1997). Reglamento general para el control de emisiones de vehículos automotores.Decreto No 32-97, La Gaceta Diario Oficial, (114), 18 de junio. Recuperado el 3 de junio de 2010, de www.fundenic.org.ni/Biblioteca/ contaminacion/Dec32-97.pdf

Asamblea Nacional. (1998). Reforma al Decreto 66-97. Decreto No. 22-98, La Gaceta, Diario Oficial, (114), 22 de abril. Recuperado el 10 de junio de 2010, de www. fundenic.org.ni/Biblioteca/contaminacion/Dec22-98.pdf

Banco Central de Nicaragua. (2010). Estadísticas económicas trimestrales y mensuales (1996-2004). Recuperado el 10 de junio de 2010, de http://www.bcn.gob.ni/ 
estadisticas/\#

Brugman, A. (2004). Diseño de un programa de instrumentos económicos para el manejo y control de la contaminación atmosférica urbana en Colombia. Ministerio de Ambiente, Vivienda y Desarrollo Territorial. Presentación realizada en la Universidad de los Andes en Bogotá-Colombia. Programa de maestrías en economía ambiental, 15 de agosto de 2006.

Castillo, J., Torres, M., \& Fernández, G. (2001). Los efectos agudos de la contaminación el aire en la salud de la población: evidencia de estudios epidemiológicos. México D.F. Recuperado el 25 de abril de 2010, de http://www.insp.mx/salud/index. html.

Centro de investigación del medio ambiente [UNI-CIEMA]. (2001). Monitoreo de la calidad de aire en la ciudad de Managua, Proyecto aire puro (1996-2001). Informe en CD. Managua: CIEMA.

Gerking, S. \& Stanley, L. (1986). Un análisis económico de la contaminación del aire y la salud: El caso de ST. Louis. Boston: North- Holland publishing Company.

Germany, B. (2003). Aspecto de salud de la contaminación del aire y el material particulado, ozono y dióxido de nitrógeno. Recuperado el 2 de julio de 2010, de http://europa. eu.int/comm/environmental/air/cafe/indx.htm.

Ibáñez, L. \& McConnell, J. (2001). Valuing Morbidity: Acute Respiratory Illnesses in Bogotá, Colombia. Paper Prepared for AERE Workshop. Recuperado el 21 de marzo de 2010, de http://www.google.com.ni/url?sa=t\& source=web\& $\mathrm{cd}=1 \&$ ved $=0 \mathrm{CB}$ QQFjAA\& url=http\%3A\%2F\%2Fwww.banrep.gov.co\%2Fdocumentos\%2Fs eminarios\%2Fword\%2FValuing\%2520Morbidity.doc\& $r$ ct=j\&q=Paper\%20 Prepared \%20 for\%20AERE\%20Workshop\%2C\%20Valuing\%20 Morbidity\%3A\%20Acute\%20Respiratory\%20Illnesses\%20in\%20 Bogot\%C3\%A1\%2C\%20Colombiakei=ImRxTM3_L8O88gax4_29Aw\&us $\mathrm{g}=\mathrm{AFQjCNGCQi3ux2gLUJkp0QIN7ZIGGzSpUg}$

Instituto Nacional de Información de Desarrollo [INIDE]. (2005). Encuesta de Medición del Nivel de Vida - EMNV. Base de datos en soporte CD. Managua: INIDE.

Instituto Nicaragüense de Energía [INE]. (2006). Consumo nacional mensual de hidrocarburos por producto y región. Recuperado el 7 de junio de 2010, de http://www.ine.gob.ni/DGH/estadisticas/2006/COMEN\%20POR\%20 REGION2006.pdf

Instituto Nicaragüense de Energía [INE]. (2007). Resumen de precios al consumidor de los combustibles no regulados, en Managua. Recuperado el 7 de junio de 2010, de http://www.ine.gob.ni/DGH/monitoreos/2008/RPP\%202007.pdf

Instituto Nicaragüense de Energía [INE]. (2008). Resumen de precios al consumidor de los combustibles no regulados, en Managua. Recuperado el 7 de junio de 2010, de http://www.ine.gob.ni/DGH/monitoreos/2008/RPP_2008.pdf

Instituto Nicaragüense de Energía [INE]. (2009). Precios promedios al consumidor de los combustibles no regulados, en Managua. Recuperado el 7 de junio de 2010, de http://www.ine.gob.ni/DGH/estadisticas/2009/totales/RPP_ENE_ DIC_2009.pdf

Ministerio de Salud. (2005). Informe sobre estadística de morbilidad en Managua 1996 2005. Soporte CD. Managua: MINSA. 
Ostro, B. (1998). Cómo estimar los efectos de la contaminación atmosférica en la salud: caso Santiago de Chile. Trabajo presentando en el seminario organizado por el Centro de Estudios Públicos, Santiago de Chile, 22 de mayo de 1997.

Parkin, M. (2001). Microeconomía versión para Latinoamérica. México: Addison Wesley. Smith, V. \& Kaoru, Y. (1990). Signals or Noise? Explaining the Variation in Recreation Benefit Estimates. American Journal of Agricultural Economics, 72 (2), 419-433.

\section{Anexo 1}

Salidas de la regresión de la curva de demanda en Stata 11.

$\begin{array}{rrrr}\text { Source | } & \text { SS } & \text { df } & \text { MS } \\ \text { Model | } & 2.35877039 & 2 & 1.17031284 \\ \text { Residual | } & 5.88710911 & 45 & 1.38685052 \\ -20679428\end{array}$

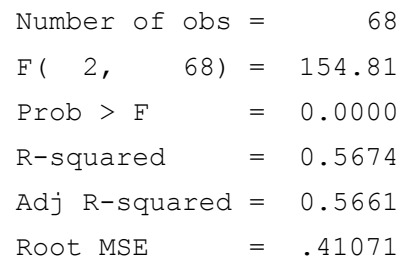

\begin{tabular}{|c|c|c|c|c|c|c|c|}
\hline Consumo & । & Coef. & Std. Err. & t & $P>|t|$ & [95\% Conf. & Interval] \\
\hline $\mathrm{P}$ & । & $5.93 e+07$ & .0029485 & 2.71 & 0.002 & $-1.06 e+07$ & 6.013789 \\
\hline $\mathrm{p} 2$ & । & $-3.18 e+07$ & .0003297 & -19.76 & 0.000 & $-3.32 e+07$ & -.0058682 \\
\hline _cons & । & $1.67 e+07$ & .0330898 & 2.29 & 0.029 & -2.492643 & 3.622419 \\
\hline
\end{tabular}

